\title{
Proteins Annexin A2 and PSA in Prostate Cancer Biopsies Do Not Predict Biochemical Failure
}

\author{
DAVID S. LAMB ${ }^{1}$, SVEN SONDHAUSS ${ }^{2}$, JONATHAN C. DUNNE ${ }^{2}$, LISA WOODS ${ }^{3}$, BRETT DELAHUNT ${ }^{4}$, \\ PETER FERGUSON $^{4}$, JUDITH MURRAY $^{1}$, JOHN N. NACEY ${ }^{5}$, JAMES W. DENHAM ${ }^{6}$ and T. WILLIAM JORDAN ${ }^{2}$ \\ ${ }^{1}$ Prostate Cancer Trials Unit, Department of Pathology and Molecular Medicine, and \\ Departments of ${ }^{4}$ Pathology and Molecular Medicine, and ${ }^{5}$ Surgery and Anaesthesia, \\ University of Otago Wellington, Wellington, New Zealand; \\ ${ }^{2}$ Centre for Biodiscovery and School of Biological Sciences, and ${ }^{3}$ School of Mathematics and Statistics, \\ Victoria University of Wellington, Wellington, New Zealand; \\ ${ }^{6}$ School of Medicine and Public Health, University of Newcastle, Newcastle, Australia
}

\begin{abstract}
Background/Aim: We previously reported the use of mass spectrometry and western blotting to identify proteins from tumour regions of formalin-fixed paraffin-embedded biopsies from 16 men who presented with apparently localized prostate cancer, and found that annexin A2 (ANXA2) appeared to be a better predictor of subsequent biochemical failure than prostate-specific antigen (PSA). Materials and Methods: In this follow-up study, ANXA2 and PSA were measured using western blotting of proteins extracted from biopsies from 37 men from a subsequent prostate cancer trial. Results: No significant differences in ANXA2 and PSA levels were observed between men with and without biochemical failure. The statistical effect sizes were small, $d=0.116$ for ANXA2, and 0.266 for PSA. Conclusion: ANXA2 and PSA proteins measured from biopsy tumour regions are unlikely to be good biomarkers for prediction of the clinical outcome of prostate cancer presenting with apparently localized disease.
\end{abstract}

Serum prostate-specific antigen (PSA) is a measure widely used to assist in the early detection of prostate cancer, but on its own is of limited value in predicting the clinical course of a prostate cancer. There is therefore a need to identify biomarkers that better predict tumour behaviour and so facilitate improved individualized treatment [reviewed in (14)]. Many human studies have focused on discovery of predictive or prognostic biomarkers from tissue biopsies,

Correspondence to: Dr T. William Jordan, Centre for Biodiscovery and School of Biological Sciences, Victoria University of Wellington, PO Box 600, Wellington, New Zealand. Tel: +64 44636092, Fax: +64 44635331, e-mail: bill.jordan@vuw.ac.nz

Key Words: Prostate cancer, proteins, FFPE sections, annexin A2, PSA. plasma or serum, exosomes, and urine or prostatic secretions, including measurement of genes, transcripts, proteins and metabolites (1-10). The focus for our group has been the discovery of proteins that could be used to predict the likely behaviour of prostate cancer at the time of the initial diagnosis. Access to archival collections of formalin-fixed paraffin-embedded (FFPE) cancer specimens with associated clinical details including treatment and disease outcome provides a resource for potential discovery of biomarkers, and we have therefore used archival specimens collected as part of the Trans-Tasman Radiation Oncology Group (TROG) trials of radiation plus androgen deprivation for treatment of locally advanced prostate cancer $(11,12)$.

We previously reported use of mass spectrometry to identify proteins from FFPE prostate cancer tissue, followed by western blotting to show that the regulatory protein annexin A2 (ANXA2) appeared to be the best predictor of biochemical failure, which was usually due to development of metastatic disease (13). Our previous examination of prospective biomarkers was carried out using archival FFPE specimens from 16 patients from the TROG Trial 96.01 trial of radiation plus androgen deprivation (11). The TROG Trial 96.01 cases used for protein analysis were chosen to represent a range of times to biochemical failure. The current study set out to test the conclusions of the pilot study by using a new set of otherwise unselected FFPE biopsies from men entered into the subsequent TROG 03.04 trial, which was of androgen suppression and radiotherapy with or without zoledronic acid (12).

\section{Materials and Methods}

Biopsy specimens for the current work were from the Wellington cohort of men enrolled on the TROG 03.04 trial. Proteomic studies on the biopsies were approved by the Central Ethics Committee of 
New Zealand (approval number WGT/03/06/065/AM04). All men on the trial presented with locally advanced prostate cancer without evidence of distant metastases. The presenting tumour characteristics of men on the trial were as described in the first end-point report (12), when the median follow-up of trial patients was 6.5 years.

Prior to protein analysis, archival haematoxylin and eosin (H\&E)stained sections from each case were examined by a pathologist (BD, $\mathrm{PF})$ to confirm the presence of both tumour and normal regions, which were then excised from adjacent unstained sections. Sufficient protein for western blot analysis was excised from each of the tumour and control regions of 37 FFPE specimens. A further 11 blocks contained tumour tissue but insufficient normal tissue to allow both tumour and control amounts of the proteins to be quantified. For western blotting, 10-20 $\mu \mathrm{g}$ of extracted protein from each sample was subjected to sodium dodecyl sulfate-polyacrylamide gel electrophoresis (SDS-PAGE) prior to electrophoretic transfer and reaction with antibodies for protein detection.

Biochemical methods, including protein extraction from tumour and control regions from archival FFPE biopsy specimens, and use of multiplexed western blotting to measure ANXA2, PSA and actin, were as previously described (13). Briefly, deparaffinised $10 \mu \mathrm{m}$ FFPE sections were incubated in $250 \mu \mathrm{l} 40 \mathrm{mM}$ Tris- $\mathrm{HCl}(\mathrm{pH} \mathrm{8.2)}$, $2 \%$ SDS, $3 \%$ dithiothreitol for $1 \mathrm{~h}$ at room temperature followed by $20 \mathrm{~min}$ at $100^{\circ} \mathrm{C}$. Extracted proteins were precipitated at $-20^{\circ} \mathrm{C}$ overnight using a ProteoExtract ${ }^{\circledR}$ Protein Precipitation Kit (EMD Millipore, Billerica, MA, USA), then resuspended in 1xlithium dodecyl sulfate (LDS) sample buffer containing sample reducing agent (Invitrogen, Carlsbad, CA, USA) and separated on 4-12\% one-dimensional SDS-PAGE gels prior to transfer to Hybond-LFP membranes (GE Healthcare, Pittsburgh, PA, USA) for $1 \mathrm{~h}$ at $30 \mathrm{~V}$. After blocking, membranes were incubated overnight with 1:100 anti-annexin A2 (C-10, mouse monoclonal, sc-28385; Santa Cruz Biotechnology, Dallas, TX, USA) and 1:200 anti-PSA (C-19, goat polyclonal, sc-7638; Santa Cruz Biotechnology); followed by sequential incubation with the fluorophore conjugated secondary antibodies AlexaFluor ${ }^{\circledR} 555$ goat anti-mouse $\operatorname{IgG}(\mathrm{H}+\mathrm{L})$ and AlexaFluor ${ }^{\circledR} 647$ chicken anti-goat $\operatorname{IgG}(\mathrm{H}+\mathrm{L})$, both at $1: 2,500$. Actin was measured on the same membranes using a mouse monoclonal anti-actin antibody (MAB1501; Sigma-Aldrich, St. Louis, MO, USA) followed by AlexaFluor ${ }^{\circledR 555}$ goat anti-mouse IgG $(\mathrm{H}+\mathrm{L})$ 1:2500. Proteins were quantified using Image $\mathrm{J}^{\mathrm{TM}}$ software (https://imagej.net/ImageJ) after scanning the developed blots with a FLA-5100 fluorescent scanner (FujiFilm, Tokyo, Japan). ANXA2 and PSA abundances were normalized against the actin abundance on the same western blot, and ANXA2 and PSA tumour/control ratios $(\mathrm{T} / \mathrm{C})$ were calculated to correct for interindividual variation in protein amounts (13). For statistical analysis, data were log-transformed and two-sided independent samples ttests were carried out using IBM SPSS Statistics 23 (IBM Corp., Armonk, NY, USA). Power calculations were performed using $\mathrm{G}^{*}$ Power (14).

\section{Results and Discussion}

Baseline characteristics for the 37 men with sufficient FFPE tissue for protein analysis of tumour and control regions were Gleason score 7-9, $\mathrm{T}$ stage $2 \mathrm{~b}$ or above, and serum PSA 2.2-47.7 ng/ml. All men had then received external beam radiation therapy in conjunction with either 6 months (shortterm) or 18 months (intermediate-term) of androgen suppression with or without 18 months of zoledronic acid (12). Subsequently, at the time of protein analysis, the clinical follow-up of the 37 men in the sample was 9-13 years, and 12 men had experienced biochemical failure (Table I), with a median time to failure of 46 months.

Initial statistical analysis focused on comparison of mean T/C forANXA2 and PSA between patients that sustained biochemical failure and those that did not (Table II). The protein expression data were not normally distributed therefore independent samples t-tests were performed on log-transformed data. There was no significant difference in T/C for ANXA2 between patients that sustained biochemical failure and those that did not $[\mathrm{t}(35)=0.332, p=0.742]$, with only a small effect size $(d=0.116)$; nor was there a significant difference in that for PSA $[\mathrm{t}(35)=0.725, p=0.473, d=0.266]$. The effect sizes for T/C for ANXA2 and PSA support an expectation for relatively small effects. For comparison, if the effect size (15) had been large (greater than 1.012) there would have been $80 \%$ power to detect it using the current sample set of 37 cases. Alternatively, based on the current results, a sample size of at least 788 men would be required to detect a small effect $(d=0.2$ ) with $80 \%$ power using a two-sided $t$-test with $\alpha=0.05$.

One difference in design between the previous and the current study is that the previous analysis was of FFPE specimens chosen to represent a range of times to biochemical failure (five cases $>100$ months, five cases $\leq 40$ months, and six 'intermediate' of 50-87 months). That analysis of 16 men from the TROG 96.01 trial, in which 11 out of the 16 cases suffered biochemical failure within 100 months, indicated that a more than 2-fold T/C ANXA2, or 3-fold T/C for PSA predicted time to biochemical failure. For the current TROG 03.04 sample dataset, the estimated odds of biochemical failure occurring were therefore also calculated at the ANXA2 and PSA cut-offs that previously gave significant differences using Kaplan-Meier analysis (13). In the current study, only 7 out of the 37 patients $(18.9 \%$ ) had a more than 2 -fold T/C for ANXA2 and only one of these experienced biochemical failure. Patients with T/C for ANXA2 $>2$ were less likely to experience biochemical failure within the study period: odds ratio $=0.288,95 \%$ confidence interval $=0.031-2.714$. Similarly, there were 14 patients $(37.8 \%)$ with T/C for PSA of more than 3 -fold but only three experienced biochemical failure and patients with T/C PSA > 3 were also less likely to experience biochemical failure: odds ratio $=0.424,95 \%$ confidence interval $=0.092-1.953$.

The predictive ratios for ANXA2 and PSA established in the previous work were therefore not supported in this follow-up study, although the number of cases was small. Assuming only $37.8 \%$ of patients experience a more than 3 -fold T/C for PSA, a sample of at least 235 men would be required to determine there is a significant association between experiencing biochemical failure and a more than 3-fold T/C for PSA with $80 \%$ power and $\alpha=0.05$. Similarly, assuming $18.9 \%$ of patients 
Table I. Summary of cases used for protein analysis of tumour and control regions. Archival formalin-fixed paraffin-embedded (FFPE) samples were from men from the Wellington cohort of the Trans-Tasman TROG 03.04 trial of androgen suppression and radiotherapy, with or without zoledronic acid, in men with locally advanced prostate cancer (12).

\begin{tabular}{lcc}
\hline Treatment & $\begin{array}{c}\text { Number of cases with sufficient FFPE tissue } \\
\text { for calculation of protein T/C ratios }\end{array}$ & $\begin{array}{c}\text { Number of cases with } \\
\text { biochemical failure }\end{array}$ \\
\hline EBRT + STAD & 11 & 3 \\
EBRT + ITAD & 9 & 1 \\
EBRT + STAD + Z & 9 & 3 \\
EBRT + ITAD + Z & 8 & 5 \\
\hline
\end{tabular}

EBRT: External beam radiotherapy, STAD: short-term (6 months) androgen deprivation, ITAD: intermediate-term (18 months) androgen deprivation, Z: zoledronic acid.

Table II. Tumour/control (T/C) ratios for annexin A2 (ANXA2) and prostate-specific antigen (PSA) in prostate cancer biopsies from 37 men with or without subsequent biochemical failure.

\begin{tabular}{lccccc}
\hline Biochemical failure & \multicolumn{2}{c}{ Time to biochemical failure (months) } & & \multicolumn{2}{c}{ T/C, mean \pm SD } \\
\cline { 2 - 5 } & Mean \pm SD & Median & Range & & ANXA2 \\
\hline Yes $(\mathrm{n}=12)$ & $61.17 \pm 36.13$ & 45.5 & $24-120$ & $0.99 \pm 0.63$ & $2.86 \pm 4.10$ \\
No $(\mathrm{n}=25)$ & - & - & - & $1.17 \pm 0.98$ & $6.19 \pm 12.11$ \\
\hline
\end{tabular}

experience a more than 2-fold T/C for ANXA2, a sample of at least 210 men would be required to determine that there is a significant association between experiencing biochemical failure and a more than 2-fold T/C for ANXA2.

In this study of 37 men from the TROG 03.04 trial, there were no significant differences in the expression of ANXA2 or PSA in tumour samples according to whether or not men subsequently underwent biochemical failure. This can be compared to the findings of the pilot study of 16 men from the TROG 96.01 trial, which demonstrated a statistically significant association between ANXA2 and biochemical failure, and a weak association between PSA and biochemical failure (13). One difference between the studies was that the TROG 96.01 cases were chosen to represent a range of times to biochemical failure, plus sufficient excised control and tumour for western blotting of ANXA2, PSA, and actin. In contrast, in the current analysis, all available archival FFPE samples with sufficient tumour and control regions were used without selection based on disease outcome. One possible confounding factor is that expansion of the tumour mass in patients with metastatic disease (16) may mean that men with high-grade locally advanced cancer who are more likely to develop metastatic disease may have very little control tissue present in archival FFPE blocks.

The findings of this study are important although based on small sample sizes. The treatment and clinical follow-up of men on whom proteomic studies are performed needs to be standardized, and this only occurs within the prospect of a randomized controlled trial. The 37 trial subjects from a single centre with sufficient archival tissue for analysis can be considered a sufficient number for a proteomic study of this type. A much larger sample size could be achieved in a multi-centre prospective study in which extra biopsy material would be taken at the onset to ensure that the great majority of trial subjects could undergo proteomic studies to see if there were correlations with subsequent clinical outcome.

However, given that no significant differences were detected in the current work, ANXA2 or PSA protein measured in excised tissue regions are unlikely to be clinically useful prognostic biomarkers for prostate cancer diagnosed in patients with localized disease, and do not warrant the cost and effort of further investigation in a larger study.

\section{Acknowledgements}

Funding support from the Cancer Society of New Zealand is gratefully acknowledged.

\section{References}

1 Zhao L, Yu N, Guo T, Hou Y, Zeng Z, Yang X, Hu P, Tang X, Wang $\mathrm{J}$ and Liu M: Tissue biomarkers for prognosis of prostate cancer: a systematic review and meta-analysis. Cancer Epidemiol Biomarkers Prev 23: 1047-1054, 2014. 
2 Tonry CL, Leacy E, Raso C, Finn SP, Armstrong J and Pennington SR: The role of proteomics in biomarker development for improved patient diagnosis and clinical decision making in prostate cancer. Diagnostics 6: 27, 2016.

3 Gaudreau PO, Stagg J, Soulières D and Saad F. The present and future of biomarkers in prostate cancer: proteomics, genomics, and immunology advancements. Biomark Cancer 8(Suppl 2): 1533, 2016.

4 Tanase CP, Codrici E, Popescu ID, Mihai S, Enciu AM, Necula LG, Preda A, Ismail $G$ and Albulescu R: Prostate cancer proteomics: Current trends and future perspectives for biomarker discovery. Oncotarget 8: 18497-18512, 2017.

5 Sequeiros T, García M, Montes M, Oliván M, Rigau M, Colás E, de Torres I, Morote J, Reventós J and Doll A: Molecular markers for prostate cancer in formalin-fixed paraffin-embedded tissues. Biomed Res Int 2013: 283635, 2013.

6 Shipitsin M, Small C, Choudhury S, Giladi E, Friedlander S, Nardone J, Hussain S, Hurley AD, Ernst C, Huang YE, Chang H, Nifong TP, Rimm DL, Dunyak J, Loda M, Berman DM and Blume-Jensen P: Identification of proteomic biomarkers predicting prostate cancer aggressiveness and lethality despite biopsy-sampling error. Br J Cancer 111: 1201-1212, 2014.

7 O'Hurley G, Busch C, Fagerberg L, Hallström BM, Stadler C, Tolf A, Lundberg E, Schwenk JM, Jirström K, Bjartell A, Gallagher WM, Uhlén M and Pontén F: Analysis of the human prostate-specific proteome defined by transcriptomics and antibody-based profiling identifies TMEM79 and ACOXL as two putative, diagnostic markers in prostate cancer. PLoS One 10: $\mathrm{e} 0133449,2015$.

8 Kim Y, Jeon J, Mejia S, Yao CQ, Ignatchenko V, Nyalwidhe JO, Gramolini AO, Lance RS, Troyer DA, Drake RR, Boutros PC, Semmes OJ and Kislinger T: Targeted proteomics identifies liquid-biopsy signatures for extracapsular prostate cancer. Nat Commun 7: 11906, 2016.

9 Lima AR, Bastos Mde L, Carvalho M and Guedes de Pinto P: Biomarker discovery in human prostate cancer: an update in metabolomics studies. Transl Oncol 9: 357-370, 2016.

10 Aiello D, Casadonte F, Terracciano R, Damiano R, Savino R, Sindona $\mathrm{G}$ and Napoli A: Targeted proteomic approach in prostatic tissue: a panel of potential biomarkers for cancer detection. Oncoscience 8: 220-241, 2016.
11 Denham JW, Steigler A, Lamb DS, Joseph D, Turner S, Matthews J, Atkinson C, North J, Christie D, Spry NA, Tai KH, Wynne C and D'Este C: Short-term neoadjuvant androgen deprivation and radiotherapy for locally advanced prostate cancer: 10-year data from the TROG 96.01 randomised trial. Lancet Oncol 12: 451-459, 2011.

12 Denham JW, Joseph D, Lamb DS, Spry NA, Duchesne G, Matthews J, Atkinson C, Tai KH, Christie D, Kenny L, Turner S, Gogna NK, Diamond T, Delahunt B, Oldmeadow C, Attia J and Steigler A: Short-term androgen suppression and radiotherapy versus intermediate-term androgen suppression and radiotherapy, with or without zoledronic acid, in men with locally advanced prostate cancer (TROG 03.04 RADAR): an open-label, randomised, phase 3 factorial trial. Lancet Oncol 15: 1076-1089, 2014.

13 Dunne JC, Lamb DS, Delahunt B, Murray J, Bethwaite P, Ferguson P, Nacey JN, Sondhauss S and Jordan TW: Proteins from formalin-fixed paraffin-embedded prostate cancer sections that predict the risk of metastatic disease. Clin Proteomics 12: 24, 2015.

14 Faul F, Erdfelder E, Lang A-G and Buchner A: G*Power 3: A flexible statistical power analysis program for the social, behavioral, and biomedical sciences. Behav Res Methods 39: 175-191, 2007.

15 Cohen J: Statistical Power Analysis for the Behavioral Sciences. Second Edition. Hillsdale, NJ, Lawrence Earlbaum Associates, pp. 25-26, 1988.

16 Hoogland AM, Kweldam CF and van Leenders GJ: Prognostic histopathological and molecular markers on prostate cancer needle-biopsies: a review. Biomed Res Int 2014: 341324, 2014.

Received September 8, 2017

Revised October 3, 2017

Accepted October 9, 2017 